\title{
A case of retiform mycosis fungoides
}

\section{Jew OS, Cornejo CM, Spaccarelli N, Vittorio CC, Samimi S, Rook AH, Haun PL and Kim EJ}

${ }^{1}$ B.A., Perelman School of Medicine, University of Pennsylvania, Philadelphia, PA, USA

${ }^{2}$ M.D., Department of Dermatology, Perelman School of Medicine, University of Pennsylvania, Philadelphia, PA, USA

\section{Introduction}

Mycosis fungoides (MF), the most common subtype of cutaneous $\mathrm{T}$ cell lymphoma (CTCL), has been referred to as one of the "great imitators" due to the heterogeneity of its cutaneous manifestations. We present a case of a 69-year-old man with an atypical case of retiform MF.

\section{Case report}

A 69-year-old man with recently diagnosed Stage IVA1 (T2N0M0B2) folliculotropic mycosis fungoides (MF) with blood involvement presented with a one-year history of waxing and waning, asymptomatic, retiform eruption on the face. The facial eruption, which appeared clinically different from his MF lesions, would develop approximately every one to two weeks, was not associated with any triggers, and spontaneously resolved within 1-2 days. It typically presented as non-palpable, retiform, red patch on the forehead and left side of his face, though the exact location would vary with each occurrence. He intermittently applied hydrocortisone $2.5 \%$ ointment to his face during these flares with no noticeable benefit. After the initiation of a multimodality regimen of extracorporeal photopheresis (ECP), psoralen plus ultraviolet A (PUVA) therapy, interferon gamma, and oral bexarotene for his MF, the patient continued to develop this waxing and waning facial eruption over the next 2-3 months. At that point, decision was made to biopsy the eruption. Examination was significant for a non-blanching, red, retiform patch with focal areas of scaling involving the forehead, left eyebrow, and left nasolabial fold (Figure 1). Histopathology showed hyperchromatic and irregularly contoured epidermotropic lymphocytes, some of which formed collections within the epidermis-so-called "Pautrier's microabcesses." These atypical lymphocytes were also present along the dermalepidermal junction with perinuclear halos and within perifollicular
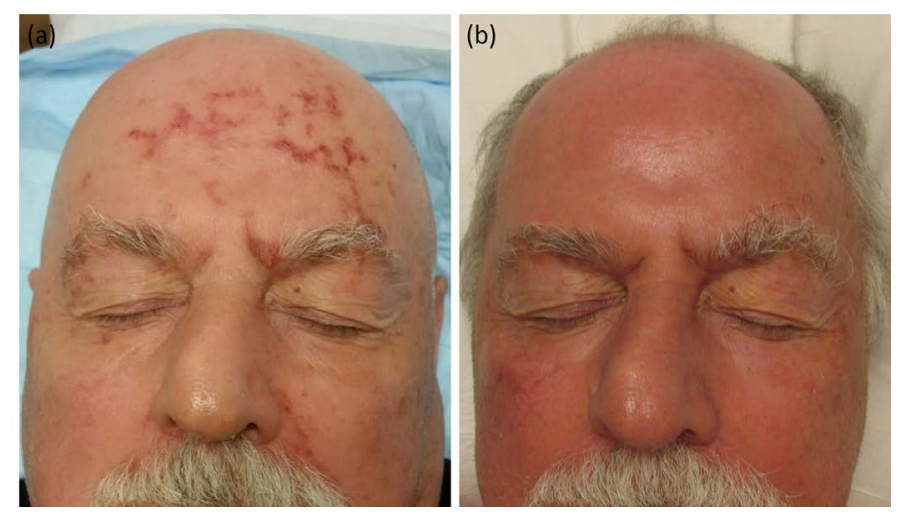

Figure 1. (a) Non-blanching, red, retiform patch involving the forehead, left eyebrow, and left nasolabial fold and (b) resolution of the retiform facial eruption after several weeks of PUVA. epithelium. These lymphocytes were of T-cell origin as highlighted by a CD3 stain; the CD4:CD8 ratio is elevated at a ratio of 15:1. Taken together, these findings are consistent with mycosis fungoides. Based on these findings, the patient was advised to expose his face, which had previously been shielded, during his PUVA sessions. This treatment resulted in sustained clearance (Figure 2).

\section{Discussion}

Mycosis fungoides (MF), the most common subtype of cutaneous $\mathrm{T}$ cell lymphoma (CTCL), is a mature $\mathrm{T}$ cell non-Hodgkin lymphoma with presentation in the skin, but with the potential to involve the lymph nodes, blood, and viscera [1]. The cutaneous manifestations of MF are heterogeneous and typically involve widespread or localized patches or plaques, tumors, and/or generalized erythroderma. MF generally exhibits an indolent clinical behavior, though in some patients temporary flares can occur in the setting of vasodilation (exercise, hot showers, etc.). The pathophysiology of this phenomenon is poorly understood [2]. In addition to the more typical presentations, many variants of MF have been reported, which have earned it the description of one of the "great imitators" [3].

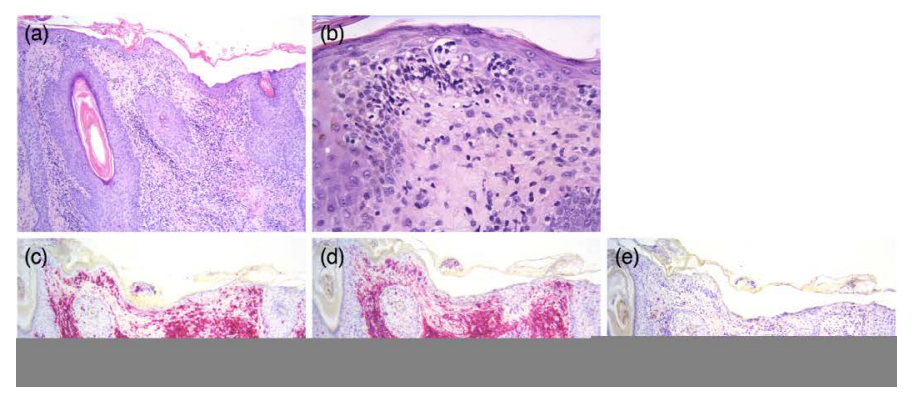

Figure 2. (a) Histologic sections illustrate increased numbers of pleomorphic T-lymphocytes with hyperchromatic and cerebriform nuclei present along the dermal-epidermal junction and perifollicular epithelium. (b) Significant epidermotropism and Pautrier'smicroabscesses are identified, along with mild dermal fibrosis. (c) A CD3 immunohistochemical stain highlights these atypical T-lymphocytes within this infiltrate. (d) and (e) The CD4:CD8 ratio is elevated at a ratio of $15: 1$. These findings are consistent with mycosis fungoides. ([a] and [b], Hematoxylin-eosin stain; original magnification [a] 100x, [b] 200x. [c], [d], and [e] Immunostain for CD3, CD4, and CD8, respectively; original magnifications: 100x).

Correspondence to: Ellen J Kim, Department of Dermatology, Dulles Building, 1st Floor, Room 1103, Hospital of the University of Pennsylvania, 3400 Spruce Street, Philadelphia, PA 19104, USA, Tel: 215-662-2397; 215-662-2737; E-mail: ellen.kim@uphs.upenn.edu

Key words: mycosis fungoides, retiform, cutaneous $T$ cell lymphoma, atypical mycosis fungoides

Received: September 05, 2016; Accepted: September 27, 2016; Published: September 30, 2016 
Zackheim and McCalmont presented a comprehensive review of various dermatoses simulated by MF, including acanthosis nigricans, alopecia, bullous eruption, comedones and epidermal cysts, dissecting cellulitis of the scalp, dyshidrosis, erythema annular centrifugum, erythema multiforme, gangrene, acquired ichthyosis, invisible dermatosis, ischemic foot, keratosis lichenoides chronica, necrobiosis, perioral dermatitis, pigmented purpuric dermatitis, pityriasis alba, porokeratosis, palmarplantar pustulosis, pyoderma gangrenosum, psoriasis, sarcoidosis, sarcoma, vesicular eruption, vesciculobullous eruption, and vitiligo [3]. In these cases, as in ours, the diagnosis of MF was not suspected clinically, but was based on histopathologic findings. Other reported forms of atypical MF include verrucous MF [4] and poikilodermatous MF [5].

Our patient, who had an otherwise typical presentation of MF on the rest of his body, suffered from recurrent flares of retiform patches on the face, which were consistent with MF on histopathology. The waxing and waning nature of his eruption may have been secondary to vasodilation, though this is not entirely clear. Although a coagulopathy and other purpuric eruptions were considered in the differential, the patient's lack of other signs and symptoms of these disorders, the histologic findings, and the sustained clearance of his eruption with PUVA treatment assisted in our diagnosis. Despite its retiform appearance, the eruption was not deemed to be vascular in origin given the presence of epidermal changes on exam and the lack of obvious vascular involvement on pathology. To our knowledge there have been no reported cases of retiform MF. This case of retiform MF highlights the importance of maintaining a high index of suspicious of atypical presentations of MF in the appropriate clinical setting.

\section{References}

1. Olsen E, Vonderheid E, Pimpinelli N, Willemze R, Kim Y, et al. (2007) ISCL EORTC. Revisions to the staging and classification of mycosis fungoides and Sezary syndrome: a proposal of the International Society for Cutaneous Lymphomas (ISCL) and the cutaneous lymphoma task force of the European Organization of Research and Treatment of Cancer (EORTC). Blood 110: 1713. [Crossref]

2. Bagherani N, Smoller BR (2016) An overview of cutaneous $\mathrm{T}$ cell lymphomas. F1000Res 5. pii: F1000 Faculty Rev-1882. [Crossref]

3. Zackheim HS, McCalmont TH (2002) Mycosis fungoides: the great imitator. $J \mathrm{Am}$ Acad Dermatol 47: 914-918. [Crossref]

4. Schlichte MJ, Talpur R, Venkatarajan S, Curry JL, Nagarajan P, et al. Verrucous presentation in patients with mycosis fungoides. Int J Dermatol 55: e126-129. [Crossref]

5. Pankratov O, Gradova S, Tarasevich S, Pankratov V (2015) Poikilodermatous mycosis fungoides: clinical and histopathological analysis of a case and literature review. Acta Dermatovenerol Alp Pannonica Adriat 24: 37-41. [Crossref]

Copyright: (C2016 Jew OS. This is an open-access article distributed under the terms of the Creative Commons Attribution License, which permits unrestricted use, distribution, and reproduction in any medium, provided the original author and source are credited. 